\title{
Cortical spreading depression and central pain networks in trigeminal nuclei modulation: time for an integrated migraine pathogenesis perspective
}

\author{
R. De Simone $\cdot$ A. Ranieri $\cdot$ S. Montella $\cdot$ \\ V. Bonavita
}

(C) Springer-Verlag Italia 2013

\begin{abstract}
The role of the cortical spreading depression (CSD)-dependent trigeminovascular activation in migraine etiopathogenesis, long considered paradigmatic, has remained substantially unproven in humans. The parallel advancement of functional neuroimaging techniques promoted the extensive exploration of the brain networks involved in pain processing in search of a possible central migraine generator. However, despite initial enthusiasms, it has not been possible to clarify whether the functional central "markers" of pain observed in primary headaches could be considered as causative or just the neural correlates of the ongoing pain. Nonetheless, our knowledge on the complex interactions between CSD, neurogenic inflammation, peripheral trigeminovascular input, central cortico-trigeminal nuclei direct modulation and pain processing and limbic system networks has enormously grown, allowing the reconceptualisation of migraine from a neurovascular to a pure neurolimbic pain disorder, therefore relocating it in the much broader frame of the brain and whole organism homeostatic control. In this work, the available evidences currently supporting the relevance of CSD, of peripheral trigeminovascular input and of direct cortico-trigeminal nuclei modulation in migraine pathogenesis are reviewed in the light of a possible integrated migraine etiopathogenetic perspective.
\end{abstract}

R. De Simone $(\bowtie) \cdot$ A. Ranieri · S. Montella

Department of Neurosciences, Headache Centre,

University "Federico II" of Naples, Via Pansini, 5,

80131 Naples, Italy

e-mail: rodesimo@unina.it

V. Bonavita

Istituto di Diagnosi e Cura Hermitage Capodimonte,

Naples, Italy
Keywords Migraine - Pathogenesis - Aura - Pain matrix · Limbic system $\cdot$ Cortical spreading depression

\section{Introduction}

The lack of evidences supporting the primary involvement of cortical spreading depression (CSD) in trigeminovascular activation has promoted, in the last decade, the extensive investigation of the brain areas involved in the processing of pain, in search of a possible causative dysfunction of these structures. These studies failed to demonstrate a definite pathology in migrainous brain but greatly expanded our knowledge on pain processing networks. Finally, recent studies on animal models reopened the question of a possible crucial role of the CSD mechanisms, at least in migraine with aura (MA). In this work, the available evidences currently supporting the relevance of CSD, of peripheral trigeminovascular input and of direct cortico-trigeminal nuclei modulation in migraine pathogenesis are reviewed, in the light of a possible integrated migraine etiopathogenetic perspective.

\section{Cortical spreading depression and migraine}

The analogies between the timing of CSD spreading and that of the progressively expanding visual aura prompted Leao, who firstly described this phenomenon in 1944 [1-3], to raise the hypothesis that CSD could be the neurophysiologic correlate of the migrainous aura. Despite the conspicuous research efforts, the first strong evidence of a causative relationship between CSD and MA only arrived in 1981 [4]. In a pivotal regional cerebral blood flow study by xenon 133 intraarterial injection, Olesen and coworkers 
demonstrated in humans the same CSD-associated perfusional changes observed in experimental models, supporting that CSD could be considered at the same time the neurophysiologic correlate of aura, and the trigger of migraine pain. In the following decade, a number of studies, largely by Moskowitz and coworkers, led to the description of the interplay between the so-called "neurogenic inflammation" and the trigeminovascular system as the basic mechanism that could explain the induction of migraine pain by CSD. After many indirect evidences, Moskowitz et al. [5] found that experimental CSD is associated with an increased ipsilateral expression of $c$-fos gene in trigeminal nuclei neurons, indicating their CSDdependent activation. In the light of the previous findings, this study supported the view that CSD is the "primary" event, leading to migraine through a neurogenic inflammation-dependent activation of peripheral trigeminal nerve endings. But Moskowitz findings raised many methodological criticisms and in 2001 were attributed to artefacts by two studies focused on the direct measuring of trigeminal, second-order neurons firing after CSD [6, 7]. In these studies, not only the trigeminal neurons activation could not be confirmed at direct recording, but neither the expected increase of plasma proteins extravasation after the CSD nor, in a parallel in vitro study, any release of CGRP (a reliable migraine attack marker [8]) could be demonstrated. This led to a substantial reconsideration of the CSD relevance in migraine etiopathogenesis [9].

\section{Does a "migraine generator" exist? The expanding knowledge on central pain networks}

Also because of the poorly substantiated applicability of the CSD-based mechanisms to migraine without aura (MO), a condition fourfold more prevalent than MA, these studies [6,7] resumed the crucial and never truly solved question of what causes the migraine attack. Rather than a specific "pathology" distinguishing migraineurs from healthy individuals, the research begun to focus on possible "functional" changes of the migrainous brain, based on the derangement of the complex network of neural structures involved in pain processing. The parallel advancement of functional neuroimaging techniques (functional magnetic resonance, positron emission tomography, voxel-based morphometry) promoted the extensive exploration of these brain structures, in search of a possible central migraine generator.

These studies contributed to the reformulation of the biological meaning of pain, by placing it in a much broader frame. Currently, we look at pain as an evolutionistically modelled, multidimensional sensation, aimed to accurately localise and discriminate the nature and the intensity of the threat to induce-also on the basis of previous experiences-the most appropriate emotional and cognitive processing of the stimulus, that could lead, in turn, to the selection of the context-specific best behavioural defensive response [10]. This new conception of pain makes it closer to other sensory modalities in which the perception of a stimulus, more or less complex, induces at the same time an emotion, a motivation and a behaviour, such as itch, temperature, sexual desire, hunger and thirst $[10,11]$.

This process requires the interplay of brain areas involved (at least) in somatic sensation, in emotion modulations, in memory and cognitive processing, in vegetative control and motor behaviour and must include inhibitory/ facilitatory mechanism for the ascending painful signals control. The so-called pain matrix [12] seems well appropriated for the goal, linking with reciprocal connections the amygdala, the thalamus, the insula cortex, the supplementary motor area, the posterior parietal cortex, the prefrontal cortex, the cingulate cortex, the periaqueductal grey, the basal ganglia, the cerebellar cortex and the primary and secondary sensory cortex. New findings from extensive functional imaging studies on central pain networks allowed the identification of patterns of cortical and brainstem activation in some way "specific" for different primary headache subtypes [12-14]. In addition, structural alterations (either as hypertrophic or hypotrophic changes) of these areas have been consistently reported in headache series $[15,16]$ as well as in other chronic pain syndromes [17-19] leading to the assumption that the repeated activation of the pain networks may promote neuroplastic cortical changes causing a permanent facilitation of pain signals, ultimately leading to the chronicisation of pain [20].

Significant achievements of these lines of research have been the development of neurostimulation techniques for pain control, ranging from the well-known deep brain stimulation (DBS) of the posterior hypothalamus in refractory chronic cluster headache [21] to the great occipital nerve stimulation [22], the repetitive transcranial magnetic stimulation [23] and the very recently proposed transcutaneal supraorbital direct current trigeminal stimulation [24]. However, despite initial enthusiasms, and even taking into account the promising results of neurostimulation studies, it has not been possible to clarify whether the functional central "markers" of primary headaches pain observed could be considered as causative factors or just the, possibly reversible [25], neural correlates of the ongoing pain [26].

From the pain matrix to the limbic system

A relevant issue which is remained long unaddressed is the possible mechanism linking migraine attacks to the many 
and heterogeneous, endogenous or exogenous triggering factors such as, among others, the physiologic premenstrual hormonal fluctuations, the prolonged sleeping or fasting, the perfumes exposure, sleep disorders, alcohol or specific food intakes and anxiety, irritability or mood changes. An intriguing unitary hypothesis that could explain how such multiple and different triggers may be involved in migraine pathophysiology preserving, at the same time, the relevance of peripheral trigeminal input, has been proposed in a study by Burstein and Jakubowski [27]. The authors observed that many different known migrainous triggers are processed in several hypothalamic nuclei and other limbic areas, all connected to the superior salivatory nucleus (SSN), an important parasympathetic station projecting, in turn, to meningeal vasculature via the sphenopalatine ganglion (SPG), and involved in the cascade of biochemical events leading to neurogenic inflammation and peripheral trigeminal activation. Since the ascending trigeminal pathways accounting for the accompanying symptoms of the migraine attack project to the same specific limbic areas (hypothalamus, midbrain, amygdala, basal forebrain) whose converging output on SSN had triggered the trigeminal vascular activation itself, a possible positive feedback loop of this articulated circuitry could account not only for the triggering but also for the perpetuation of a migraine attack for hours or days. In this light, migraine may result from a dysfunction of a wide neurolimbic pain network [28], a complex system deeply involved in homeostatic control of brain and body systems and that encompasses most of the pain matrix areas. In this integrated perspective, the peripheral trigeminovascular system could account for only a part of the whole interplay and might not be sufficient to migraine generation.

\section{New evidences on the cortical spreading depression role in migraine triggering}

Several years after the drastic reconsideration of the CSD role in migraine attack generation, two works by Burstein and Jakubowski have reopened the question. In the first study [29], an experimentally induced CSD in anesthetized rats led to a prolonged increase in meningeal nociceptor firing rate that began $14 \mathrm{~min}$ after the CSD wave. The finding demonstrates for the first time that the induction of CSD by focal stimulation of the rat visual cortex can lead to a long-lasting activation of nociceptors that innervate the meninges. The second study [30] provided evidence in rats of a sustained CSD-dependent activation of trigeminal nuclei (C1-C2) neurons in the superficial (I and II) but not in deeper laminae. Notably, I and II laminae neurons were the same in which Moskowitz et al. [5] had found an increased $c$-fos expression ipsilateral to CSD. On the contrary, the subsequent negative studies $[6,7]$ that had challenged Moskowitz findings, mainly involved the III and IV laminae trigeminal neurons. Based on these new data, that could also explain the reasons for the previous study negative findings, the crucial role of CSD as a sufficient migraine pain trigger, at least in MA, has been fully rehabilitated [31].

However, the assumption that CSD-dependent peripheral trigeminovascular activation is the main pathway involved in the second-order trigeminal neurons activation has been challenged by a very interesting work by Lambert et al. [32]. The direct recording of trigeminal nuclei neurons involved in meningeal pain processing following CSD induction showed only minor differences in firing pattern before versus after the peripheral exclusion of trigeminal input by lignocaine injection in Gasser ganglion, confirming that CSD could directly modulate the trigeminal nuclei activity. Based on these data Lambert proposed that an ordinary trigeminal traffic ("noise" signals) continuously occurs in second-order trigeminal neurons and it is actively kept below the perceivability threshold by descending modulating inputs. CSD triggering of migraine pain may therefore rely on a direct CSD-dependent disinhibition of the trigeminal nuclei, a process not necessarily requiring the peripheral trigeminal arm involvement.

Although this study raised deep criticism for a possible suboptimal study design [33], two direct anatomical topdown projections to trigeminal nuclei have been very recently described [34]. The first, associated with facilitatory effects, directly links a specific insula area to trigeminovascular neurons located in I and II laminae. The second, with inhibitory effects, originates from a specific area of the primary sensory cortex and projects to trigeminovascular neurons of the III and IV laminae. Interestingly, these connections were activated by meningeal-driven pain and not by cutaneous stimulation. This study provides the basis for a possible direct central top-down triggering of the migraine pain, at least in part independent by the peripheral trigeminal arm involvement, possibly mediated by the recurrent suppression of a central inhibitory modulation of trigeminal nuclei neurons and ultimately leading to the transmission of even ordinary and usually not perceivable trigeminal "noise" signals. This perspective fully complies with the concept that, besides the countless research efforts, no evidence has ever been found supporting a defined "pathology" distinguishing migraine from healthy individuals [35].

The pathogenetic relevance of the peripheral trigeminovascular pain structures versus the central facilitation (or the lack of descending inhibition) of afferent trigeminal signals, remains to be clarified [36]. However, on the basis of the above reported considerations and findings, it has been proposed [33] that the initial activation of second-order 
trigeminal neurons may rely on peripheral input from meningeal nociceptors, whereas the intensity and the duration of central trigeminal neurons activity could be dependent on a direct central modulation descending from different cortical and brainstem areas.

\section{An integrated migraine pathogenesis perspective}

So, what causes migraine? Despite decades of intense research in the field, we cannot yet answer to the fundamental question of what causes the migraine attack. Still, our knowledge on the complex interactions between CSD, peripheral trigeminovascular input, central trigeminal neurons modulation, pain processing and limbic system networks has enormously grown allowing the reconceptualisation of migraine from a neurovascular to a pure neurolimbic pain disorder, therefore, relocating it in the much broader frame of the brain and whole organism homeostatic control.

Following this perspective, and taking into account, on one side, the "lack of pathology" emerging from more than two decades of research efforts and, on the other, the probable evolutionary advantages [37] that migraine should have provided (not only migraine has not been eliminated by natural selection but, on contrary, it appears to slowly expand its prevalence in general population [38, 39]), we would mention a fascinating perspective put forward by Montagna et al. [40]. In this very interesting reconceptualisation of primary headaches, the inextricable puzzle represented by the multifaceted clinical characteristics of the migraine pain attack can be solved considering its striking similarity with the innate, pan-mammalian, adaptive behavioural response to visceral "unescapable" pain represented by the sickness behaviour [41]. This includes the avoidance of food intakes, of food or sexual searching behaviours and the inescapable need for a quite place where to lay or to sleep, a pattern of events fully matching the migraine-associated behaviours, emotions and symptoms. In this light, the clinical complexity of the migraine attack presentation could reflect the "physiologic" allostatic response to internal or external stimuli perceived (more or less inappropriately in migraineurs) as a body homeostasis threat to promote the homeostatic imbalance restoration through a temporary retire of the individual from any environmental interaction, including that arising from ordinary daily life activities.

Montagna and coworkers conceptualisation is now supported by the very intriguing findings of a recent study that aimed to unveil the final thalamo-cortical projection of dural sensitive neurons. Using a technique that combines single-unit recording with iontophoretic administration of an anterograde tracer into trigeminovascular-sensitive thalamic neurons, the authors found that these neurons project in multiple directions to many cortical areas involved in different brain functions, such as regulation of affect, motor capacity, visual and auditory perception, spatial orientation, memory retrieval, and olfaction [42], therefore, accounting for the perplexing diversity of neurological disturbances associated with migraine headache. Thus, in the Montagna et al.'s finalistic adaptive view of migraine, the stereotyped but enigmatic array of symptoms, signs, emotions and behaviours that characterise the migraine attack could rely on an ancient, evolutionary modelled, anatomical network that aimed to promote the mammalians sickness behaviour in response to brain, or whole organism, homeostatic balance threat.

This integrated perspective could require the reformulation of the goals of genetic and clinical research in primary headaches aetiology, that might be relocated within promoters of the periodic pain recurrence rather than into the pathogenetic dynamics of the attack itself [43]. The extraordinary prevalence of migraine, associated with its possibly ongoing epidemiological expansion, allows postulating the existence of evolutionary advantages of migraine [37]. In this light, migraine (and probably other primary headaches) could be seen as necessary, but evolutionistically affordable, biological costs [43].

Conflict of interest The author certify that there is no actual or potential conflict of interest in relation to this article.

\section{References}

1. Leao AAP (1944) Spreading depression of activity in the cerebral cortex. J Neurophysiol 7:359-390

2. Leao AAP (1944) Pial circulation and spreading depression of activity in the cerebral cortex. J Neurophysiol 7:391-396

3. Leao AAP, Morison RS (1945) Propagation of spreading cortical depression. J Neurophysiol 8:33-45

4. Olesen J, Larsen B, Lauritzen M (1981) Focal hyperemia followed by spreading oligemia and impaired activation of $\mathrm{rCBF}$ in classic migraine. Ann Neurol 9:344-352

5. Moskowitz MA, Nozaki K, Kraig RP (1993) Neocortical spreading depression provokes the expression of C-fos proteinlike immunoreactivity within the trigeminal nucleus caudalis via trigeminovascular mechanisms. J Neurosci 13(3):1167-1177

6. Lambert GA, Michalicek J, Storer RJ et al (1999) Effect of cortical spreading depression on activity of trigeminovascular sensory neurons. Cephalalgia 19:631-638

7. Ebersberger A, Schaible HG, Averbeck B et al (2001) Is there a correlation between spreading depression, neurogenic inflammation, and nociception that might cause migraine headache? Ann Neurol 49:7-13

8. Goadsby PJ, Edvinsson L, Ekman R (1990) Vasoactive peptide release in the extracerebral circulation of humans during migraine headache. Ann Neurol 28:183-187

9. Goadsby PJ (2001) Migraine, aura, and cortical spreading depression: why are we still talking about it? Ann Neurol 49:4-6

10. Craig AD (2003) A new view of pain as a homeostatic emotion. Trends Neurosci 26:303-307 
11. Bussone G, Grazzi L, Panerai AE (2012) Pain, emotion, headache. Headache 52(S2):98-101

12. May A (2006) A review of diagnostic and functional imaging in headache. J Headache Pain 7:174-184

13. Weiller C, May A, Limmroth V et al (1995) Brain stem activation in spontaneous human migraine attacks. Nat Med 1:658-660

14. Morelli N, Rota E, Gori S et al (2013) Brainstem activation in cluster headache: an adaptive behavioural response? Cephalalgia (Epub ahead of print). doi:10.1177/0333102412474505

15. Kim JH, Suh SI, Seol HY et al (2008) Regional grey matter changes in patients with migraine: a voxel-based morphometry study. Cephalalgia 28:598-604

16. DaSilva AF, Granziera C, Snyder J et al (2007) Thickening in the somatosensory cortex of patients with migraine. Neurology 69:1990-1995

17. Schmidt-Wilcke T, Leinisch E, Ganssbauer S et al (2006) Affective components and intensity of pain correlate with structural differences in gray matter in chronic back pain patients. Pain 125:89-97

18. May A (2008) Chronic pain may change the structure of the brain. Pain 137:7-15

19. Frøkjær JB, Bouwense SA, Olesen SS et al (2012) Reduced cortical thickness of brain areas involved in pain processing in patients with chronic pancreatitis. Clin Gastroenterol Hepatol 10(4):434-438

20. Sprenger T, Borsook D (2012) Migraine changes the brain: neuroimaging makes its mark. Curr Opin Neurol 25:252-262

21. Leone M, Franzini A, Proietti Cecchini A et al (2010) Hypothalamic deep brain stimulation in the treatment of chronic cluster headache. Ther Adv Neurol Disord 3:187-195

22. Silberstein S, Dodick D, Saper J et al (2012) Safety and efficacy of peripheral nerve stimulation of the occipital nerves for the management of chronic migraine: results from a randomized, multicenter, double-blinded, controlled study. Cephalalgia 32: $1165-1179$

23. André-Obadia N, Mertens P, Gueguen A et al (2008) Pain relief by rTMS: differential effect of current flow but no specific action on pain subtypes. Neurology 71:833-840

24. Schoenen J, Vandersmissen B, Jeangette S et al (2013) Migraine prevention with a supraorbital transcutaneous stimulator: a randomized controlled trial. Neurology 80:697-704

25. Grazzi L, Chiapparini L, Ferraro S et al (2010) Chronic migraine with medication overuse pre-post withdrawal of symptomatic medication: clinical results and FMRI correlations. Headache 50:998-1004

26. Borsook D, Burstein R (2012) The enigma of the dorsolateral pons as a migraine generator. Cephalalgia 32:803-812
27. Burstein R, Jakubowski M (2005) Unitary hypothesis for multiple triggers of the pain and strain of migraine. J Comp Neurol 493:9-14

28. Maizels M, Aurora S, Heinricher M (2012) Beyond neurovascular: migraine as a dysfunctional neurolimbic pain network. Headache 52:1553-1565

29. Zhang X, Levy D, Noseda R et al (2010) Activation of meningeal nociceptors by cortical spreading depression: implications for migraine with aura. J Neurosci 30:8807-8814

30. Zhang X, Levy D, Kainz V et al (2011) Activation of central trigeminovascular neurons by cortical spreading depression. Ann Neurol 69:855-865

31. Pietrobon D, Moskowitz MA (2013) Pathophysiology of migraine. Annu Rev Physiol 75:365-391

32. Lambert GA, TruongmL Zagami AS (2011) Effect of cortical spreading depression on basal and evoked traffic in the trigeminovascular sensory system. Cephalalgia 31:1439-1451

33. Burstein R, Strassman A, Moskowitz AM (2012) Can cortical spreading depression activate central trigeminovascular neurons without peripheral input? Pitfalls of a new concept. Cephalalgia 32:509-511

34. Noseda R, Constandil L, Bourgeais L et al (2010) Changes of meningeal excitability mediated by corticotrigeminal networks: a link for the endogenous modulation of migraine pain. J Neurosci 30:14420-14429

35. Lambert GA (2010) The lack of peripheral pathology in migraine headache. Headache 50:895-908

36. Goadsby PJ (2005) Migraine pathophysiology. Headache 45(Suppl 1):S14-S24

37. Loder E (2002) What is the evolutionary advantage of migraine? Cephalalgia 22:624-632

38. Sillanpaa M, Anttila P (1996) Increasing prevalence of headache in 7-year-old schoolchildren. Headache 36:466-470

39. Rozen TD, Swanson JW, Stang PE et al (2000) Increasing incidence of medically recognized migraine headache in a US population. Headache 40:224-230

40. Montagna P, Pierangeli G, Cortelli P (2010) The primary headaches as a reflection of genetic darwinian adaptive behavioural responses. Headache 50:273-289

41. Tizard I (2008) Sickness behavior, its mechanisms and significance. Anim Health Res Rev 9:87-99

42. Noseda R, Jakubowski M, Kainz V et al (2011) Cortical projections of functionally-identified thalamic trigeminovascular neurons: implications to migraine headache and its associated symptoms. J Neurosci 31:14204-14217

43. Bonavita V, De Simone R (2011) Pain as an evolutionary necessity. Neurol Sci 32(Suppl 1):S61-S66 